\title{
Bayesian Vessel Extraction for Planning of Radiofrequency-Ablation
}

\author{
Stephan Zidowitz ${ }^{1}$, Johann Drexl ${ }^{1}$, Tim Kröger ${ }^{2}$, Tobias Preusser ${ }^{2}$, \\ Felix Ritter ${ }^{1}$, Andreas Weihusen ${ }^{1}$, Heinz-Otto Peitgen ${ }^{1}$ \\ ${ }^{1} \mathrm{MeVis}$ Research GmbH, Universitätsallee 29, 28359 Bremen \\ ${ }^{2} \mathrm{CeVis}$ - Center for Complex Systems and Visualization, Department of Mathematics \\ and Computer Science, University of Bremen, Universitätsallee 29, 28359 Bremen \\ Email: zidowitz@mevis.de
}

\begin{abstract}
The software-assisted planning of radiofrequency-ablation of liver tumors calls for robust and fast methods to segment the tumor and surrounding vascular structures from clinical data to allow a numerical estimation, whether a complete thermal destruction of the tumor is feasible taking the cooling effect of the vessels into account. As the clinical workflow in radiofrequency-ablation does not allow for time consuming planning procedures, the implementation of robust and fast segmentation algorithms is critical in building a streamlined software application tailored to the clinical needs. To suppress typical artifacts in clinical CT or MRT data - like inhomogeneous background density due to the imaging procedure - a Bayesian background compensation is developed, which subsequently allows a robust segmentation of the vessels by fast threshold based algorithms. The presented Bayesian background compensation has proven to handle a wide range of image perturbances in MRT and CT data and leads to a fast and reliable identification of vascular structures in clinical data.
\end{abstract}

\section{Introduction}

To assist the complete thermal destruction of the tumor in radiofrequencytherapy a software-assisted image based planning of the ablation must consider the extension of the tumor as well as the vascular structures in the vicinity of the tumor, to incorporate the cooling effect of the vessels. Hence the softwareassisted planning of radiofrequency-ablation of liver tumors calls for robust and fast methods to segment the tumor and the surrounding vascular structures from clinical data. However, the use of fast threshold based algorithms is prevented by inhomogeneous image intensities present in clinical CT and MRT data. A reliable reduction of this image perturbances subsequently speeds up the segmentation procedures and leads to a fast and robust one-click segmentation of relevant anatomical structures. As the clinical workflow in radiofrequency-ablation does not allow for time consuming planning procedures, the implementation of robust and fast segmentation algorithms is critical in building a streamlined software application tailored to the clinical needs. 


\section{State of the art and new contribution}

The problem of removing intensity nonuniformity from MRT images has been extensively addressed by many researchers [1]. Beside homomorphic unsharp masking and other filtering techniques [2] the problem of nonuniformity is addressed using an approach becoming known under the name nonparametric nonuniform intensity normalization [3]. In this a gain field is estimated to sharpen the histogramm of the MRT data. Other researchers look uppon the intensity correction as intrinsic part of the enclosing classification and segmentation problem [4]. Commonly these algorithms for the inhomogeneity correction in MRT data rely on the estimation of multiplicative correction factors, whereas the method presented here is based on an additive two-component model. Comparable methods are used for background compensation in astrophysics [5, 6]. The proposed method has proven suitable to handle a wide range of image perturbances in MRT and CT data likewise. While we exploit the local intensity only, the statistical model provides in addition an easy to use framework to incorporate more sophisticated image measures if needed.

\section{Methods}

The widespread problem of separating vascular structures from parenchymal background in inhomogeneous clinical CT or MRT images is solved with Bayesian probability theory. To capture the defining characteristics of the images - namely that the parenchymal regions are smoother than the vascular structures - a two-component mixture model is used. Given the MRT image intensity $\left\{y_{i}\right\}$ at each voxel $i$, our complemetary hypotheses for the measurement process are

$$
\begin{array}{ll}
(\mathcal{B}) y_{i} \text { is purly background: } & y_{i}=b_{i}+\epsilon_{i} \\
(\mathcal{S}) y_{i} \text { contains signal contribution: } & y_{i}=b_{i}+s_{i}+\epsilon_{i}
\end{array}
$$

While the first hypothesis specifies that the image intensity consists only of background $b_{i}$ spoiled with noise $\epsilon_{i}$, the complementary hypothesis specifies the case where additional signal intensity $s_{i}$ contributes to the image. An additional assumption is that the background is smoother than the signal. This is enforced by approximating the data by a linear combination of smooth basis functions, namely by modelling the background using B-spline approximation of the data. Spline approximation incorporating smoothness constraints is superior to filtering techniques in dealing with given situation of data inhomogenities with a wide range of spacial scales [7]. As the spline approximation are used as filter for this application, the smoothing parameters of the B-splines - primarily the distance between basis functions - are not derived from the data but choosen a priory.

The image noise $\left\{\epsilon_{i}\right\}$ is approximated with Gaussian or Poisson statistic

$$
\begin{array}{ll}
\text { Gaussian: } & p\left(y_{i} \mid \xi_{i}\right)=\left(2 \pi \sigma^{2}\right) \exp \left[-\frac{\left(\xi_{i}-y_{i}\right)^{2}}{2 \sigma^{2}}\right] \\
\text { Poisson: } & p\left(y_{i} \mid \xi_{i}\right)=\frac{y_{i}^{\xi_{i}}}{\xi_{i} !} \exp \left(-y_{i}\right)
\end{array}
$$


where the expected value $\xi_{i}$ is given by the background $\xi_{i}=b_{i}$ for $\mathcal{B}$ or by the background with additional signal contribution $\xi_{i}=b_{i}+s_{i}$ for hypothesis $\mathcal{S}$ respectively.

The signal contribution is descripted probablistically in terms of its prior distribution. Assuming, we know only the average value $\lambda$ of the signal intensity, the prior distribution is given by an exponential function (for positive signal intensities)

$$
p\left(y_{i} \mid \lambda\right)=\frac{\exp \left[-\frac{y_{i}}{\lambda}\right]}{\lambda}
$$

Using this prior, the likelihood for the hypothesis " $(\mathcal{S}) y_{i}$ contains signal contribution" is obtained by marginalizing the noise probability over the signal. For positive signal intensities this leads to

$$
\begin{array}{ll}
\text { Gaussian: } & p_{(\mathcal{S})}\left(y_{i} \mid b_{i}, \lambda\right)=\frac{1}{2 \lambda}\left\{1+\operatorname{erf}\left[\frac{\lambda\left(y_{i}-b_{i}\right)-\sigma^{2}}{\lambda \sqrt{2 \sigma^{2}}}\right]\right\} \exp \left[\frac{-2 \lambda\left(y_{i}-b_{i}\right)+\sigma^{2}}{2 \lambda^{2}}\right] \\
\text { Poisson: } & p_{(\mathcal{S})}\left(y_{i} \mid b_{i}, \lambda\right)=\frac{\exp \left[\frac{b_{i}}{\lambda}\right]}{\lambda\left(1+\lambda^{-1}\right)^{y_{i}+1}} \frac{\Gamma\left[\left(y_{i}+1\right), b_{i}\left(1+\lambda^{-1}\right)\right]}{\Gamma\left[y_{i}+1\right]}
\end{array}
$$

where $\Gamma[a, x]$ is the incomplete Gamma-function and $\Gamma[a]=\Gamma[a, 0]$. The extension to negative signale intensities is straightforward.

For the mixture model the prior probability for the two complementary hypotheses is chosen to be independent of the localization: $p_{(\mathcal{B})}=\beta$ and $p_{(\mathcal{S})}=$ $1-\beta$.

While the background intensities $\left\{b_{i}\right\}$ are calculated using spline approximation of the data, the probility parameters $\sigma, \lambda$, and $\beta$ are estimated by histogram analysis of the remaining image intensities. Subsequently for each voxel the probability of not being background is calculated. Afterwards, a fast threshold based region growing algorithm is used on this probability map to segment the vascular structures.

\section{Results}

Combining a threshold based region growing algorithm with a Bayesian background compensation, we were able to implement a robust one-click segmentation of vascular structures for clinical CT and MRT data of the liver. The background compensation is based on two successive steps: First the background intensity variations are approximated as illustrated in figure 1. Taking the induced shift of the intensities into account, the probility parameters $\sigma, \lambda$, and $\beta$ are extracted by fitting the modelized intensity distribution to the histogram of the remaining image intensities. Figure 2 exemplifies the gain for the archivable segmentation result. The presented combination of the developed statistical data analysis with a simple threshold based region growing ensures a robust and fast vascular segmentation with results comparable to data segmentations with more sophisticated, time consuming algorithms. 
Fig. 1. Left: Slice of clinical MRT-data of the liver with typical data inhomogeneties; Right: Corresponding slice of the smoothed background intensity calculated by spline approximation (MRI is courtesy of Prof. Broelsch, University Hospital Essen)

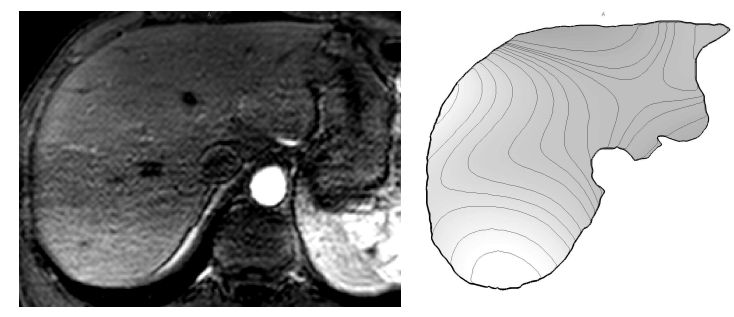

Fig. 2. Vascular structure segmented by region growing algorithm with optimized global threshold: Segmentation based on original MRT-data (left); Corresponding object segmented from probability map resulting from the bayesian data analysis (right)
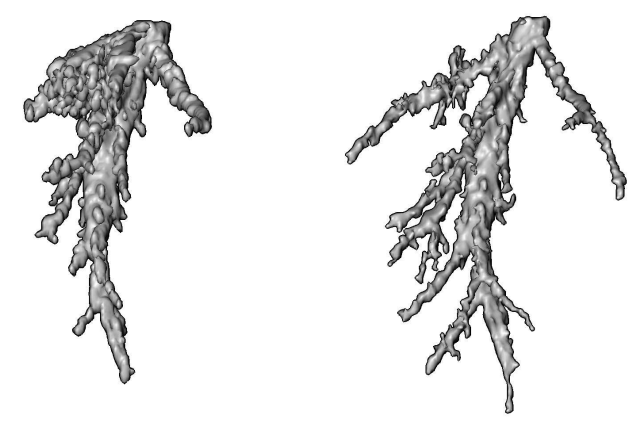

\section{Discussion}

The presented Bayesian background compensation has proven to handle a wide range of image perturbances in MRT and CT data and leads to a fast and reliable identification of vascular structure in clinical data. The presented background compensation is robust, fully automatic, and requires litte image specific knowledge. Hence it is attractive as a preprocessing step for further data analysis. Moreover, the statistical model provides an easy to use framework to incorporate more sophisticated image measures if needed. The incorporation of this algorithm improves the robustness and speed of the segmentation algorithms. This robust segmentation is a mandatory part of the carefully tailored workflow for the software-assisted patient individual planning of radiofrequency-ablation of liver tumors.

Bundling the presented vascular segmentation with a robust tumor segmentation [8], an interactive positioning of a virtual applicator-model and a numerical estimation of the region destroyed by the induced thermal energy, a clinical applicable software-assistant for the patient individual planning of radiofrequencyablation is developed [9]. Extracting anatomical information about the tumor 
and the close by vascular structures from clinical data, the implemented softwareassistant allows to approximate the effect of the radiofrequency-ablation taking the cooling effect of local vessels into account [10]. Tailored to the clinical needs, this application makes a patient individual planning of the radiofrequencyablation available in a clinical feasible workflow. Thereby the developed softwareassisted patient individual planning reduces uncertainty in the planned applicator positioning and supports the evaluation of the achievable thermal destruction. This enhances the confidence in achieving a complete thermal destruction of the tumor.

\section{Acknowledgement}

The research leading to this publication has been supported by the German Federal Ministry of Education and Research under grant number 01EZ0010 and 01BE03C as part of the cooperation projects VICORA and FUSION.

\section{References}

1. Arnold JB, Liow JS, Schaper KA, et al. Qualitative and quantitative evaluation of six algorithms for correcting intensity nonuniformity effects. Neuroimage 2001;13:931-943.

2. Brinkmann BH, Manduca A, Robb RA. Optimized homomorphic unsharp masking for MR grayscale inhomogeneity correction. IEEE Trans Med Imaging 1998;17:161171.

3. Sled JG, Zijdenbos AP, Evans AC. A nonparametric method for automatic correction of intensity nonuniformity in MRI data. IEEE Trans Med Imaging 1998;17:8797.

4. Shattuck DW, Sandor-Leahy SR, Schaper KA, et al. Magnetic resonance image tissue classification using a partial volume model. Neuroimage 2001;13:856-876.

5. Fischer R, Hanson KM, V Dose V, von Der LindenW. Background estimation in experimental spectra. Phys Rev E Stat Phys Plasmas Fluids Relat Interdiscip Topics 2000 Feb ;61 (2):1152 -60 2000;61:1152-1160.

6. Guglielmetti F, Fischer R, Dose V. Mixture modeling for background and sources separation in x-ray astronomical images. American Institut of Physics; 2004. 111118.

7. Schumaker LL, Utreras FI. On generalized cross validation for tensor smoothing splines. SIAM J Sci Stat Comput 1990;11(4):713-731.

8. Bornemann L, Kuhnigk JM, Dicken V, et al. OncoTREAT - A software assistant for oncological therapy monitoring. Procs CARS 2005; 429-434.

9. Weihusen A, Ritter F, Pereira P, et al. Towards a workflow-oriented software assistance for the radiofrequency ablation. Lecture Notes in Informatics 2006;93:507513.

10. Kröger T, Altrogge I, Preusser T, et al. Numerical simulation of radio frequency ablation with state dependent material parameters in three space dimensions. LNCS 2006;4191:380-388. 